\title{
Phonon Signatures of a Quantum Impurity with Induced Electron Pairing
}

\author{
J. BARAŃSKI AND T. DOMAŃSKI
}

Institute of Physics, M. Curie-Skłodowska University, pl. M. Curie-Skłodowskiej 1, 20-031 Lublin, Poland

\begin{abstract}
We analyze the effective spectrum of a vibrating molecule coupled between one conducting and another superconducting electrode. The proximity effect induces electron pairing which is manifested by the subgap quasiparticle peaks (the Andreev states) whose broadening depends on a hybridization with the conducting electrode. On the other hand, the electron-phonon interaction leads to a multilevel structure with the polaronic states separated by the phonon energy. We inspect a combined effect of both these (polaronic and induced pairing) phenomena.
\end{abstract}

DOI: 10.12693/APhysPolA.126.A-73

PACS: 73.63.Kv; 73.23.Hk; 74.45.+c; 74.50.+r; 63.22.-m

\section{Introduction}

Influence of the boson degrees of freedom (such as phonons or photons) on the electron tunneling through hybrid structures with the superconducting reservoirs has been initiated already half a century ago [1]. Presently similar issues attract a renewed attention in the theoretical and experimental studies of nanoscopic devices, where the quantum dots, quantum wires and/or molecules are subjected between various reservoirs of the itinerant electrons $[2,3]$. Novel fabrication techniques of the hybrid structures with the superconducting electrodes connected to non-superconducting nanostructures allow a fully controllable investigation of the competition between electron pairing and strong Coulomb repulsion [4]. Their interdependence can be also explored taking into account the bosonic vibrational modes, which contribute additional spectroscopic features [5-7].

In this report we study the single particle spectrum of a vibrating quantum dot (QD) coupled between the superconducting $(\mathrm{S})$ and metallic $(\mathrm{N})$ leads. Electronphonon interaction converts the initial QD level into a series of polaronic states, separated by the phonon energy. On the other hand, hybridization with the superconducting electrode allows the Cooper pairs to penetrate this nanoobject bringing some features of the superconducting spectrum (due to the induced electron pairing). We investigate how this induced pairing affects the spectrum of a vibrating molecule and find that the polaronic states appear simultaneously above and below the Fermi energy.

In Sect. 2 we introduce the microscopic model of our system and discuss some formal aspects of the adopted methods. To determine the spectral function of a vibrating QD we disentangle the bosonic from fermionic degrees of freedom using the Lang-Firsov canonical transformation. In last section we inspect spectroscopic fingerprints of the phononic modes of the proximized quantum dot.

\section{Vibrating quantum dot}

To describe a vibrating quantum dot hybridized with two external leads we use the Anderson-type Hamiltonian

$$
H=\sum_{\beta} H_{\beta}+H_{\mathrm{mol}}+H_{\mathrm{T}}
$$

The first terms describe the normal $(\beta=\mathrm{N})$ and superconducting $(\beta=\mathrm{S})$ electron reservoirs. We express them in the usual way by $H_{\mathrm{N}}=\sum_{\boldsymbol{k} \sigma} \xi_{\mathrm{N} \boldsymbol{k}} C_{\mathrm{N}, \boldsymbol{k} \sigma}^{\dagger} C_{\mathrm{N}, \boldsymbol{k} \sigma}$ and $H_{\mathrm{S}}=$ $\sum_{\boldsymbol{k} \sigma} \xi_{\mathrm{S} \boldsymbol{k}} C_{\mathrm{S}, \boldsymbol{k} \sigma}^{\dagger} C_{\mathrm{S}, \boldsymbol{k} \sigma}-\sum_{\boldsymbol{k}}\left(\Delta^{*} C_{\mathrm{S} \boldsymbol{k} \downarrow} C_{\mathrm{S}-\boldsymbol{k} \uparrow}+\Delta C_{\mathrm{S}-\boldsymbol{k} \uparrow}^{\dagger} C_{\mathrm{S} \boldsymbol{k} \downarrow}^{\dagger}\right)$, where $C_{\beta \boldsymbol{k} \sigma}^{(\dagger)}$ denote the annihilation (creation) operators for spin $\sigma$ electrons with momentum $\boldsymbol{k}$ and energy $\xi_{\beta} \boldsymbol{k}$ (measured with respect to the chemical potential $\mu_{\beta}$ ). The energy gap of superconductor is denoted by $\Delta$. The vibrating molecule is described by the following Hamiltonian:

$$
\begin{aligned}
& H_{\mathrm{mol}}=\sum_{i, \sigma} \epsilon d_{\sigma}^{\dagger} d_{\sigma}+U n_{\uparrow} n_{\downarrow}+\omega_{0} a^{\dagger} a \\
& \quad+\lambda \sum_{\sigma} d_{\sigma}^{\dagger} d_{\sigma}\left(a^{\dagger}+a\right),
\end{aligned}
$$

where $d_{\sigma}^{(\dagger)}, d_{\sigma}$ are the second quantization operators for the QD electron with spin $\sigma$ and $a^{(\dagger)}$ correspond to the monochromatic phonon mode of energy $\omega_{0}$. The initial QD level is denoted by $\epsilon$ and the corresponding electronboson coupling by $\lambda$. As usually $U$ is the Coulomb potential energy. Hybridization of the molecule with the external leads is represented by

$$
H_{T}=\sum_{\beta=N, S} \sum_{\boldsymbol{k} \sigma} V_{\beta, \boldsymbol{k} \sigma} C_{\beta, \boldsymbol{k} \sigma}^{\dagger} d_{\sigma}+\text { H.c. }
$$

To determine the single-particle Green function $G(\omega)$, expressed in the $2 \times 2$ Nambu spinor representation [8], we use the Dyson equation

$$
G^{-1}(\omega)=g_{\text {mol }}^{-1}(\omega)-\Sigma_{\mathrm{N}}(\omega)-\Sigma_{\mathrm{S}}(\omega),
$$

where $g_{\mathrm{mol}}(\omega)$ is the propagator of the isolated (but vibrating) molecule and the selfenergies $\Sigma_{\mathrm{N}}, \Sigma_{\mathrm{S}}$ describe the hybridization effects. For clarity reasons we shall neglect the influence of mutual electron interactions (which would additionally cause the Coulomb blockade and can induce the low energy feature in the Kondo regime [9]).

The hybridization $H_{\mathrm{T}}$ contributes the following selfenergies [8]:

$$
\Sigma_{\beta}(\omega)=\sum_{\boldsymbol{k}} V_{\beta, \boldsymbol{k}} g_{\beta}(\boldsymbol{k}, \omega) V_{\beta, \boldsymbol{k}}^{*},
$$


where $g_{\beta}(\boldsymbol{k}, \omega)$ are the Green functions of the external electrodes, which have the standard form [9]:

$$
\begin{aligned}
& g_{\mathrm{N}}(\boldsymbol{k}, \omega)=\left(\begin{array}{cc}
\frac{1}{\omega-\xi_{\boldsymbol{k}, \beta}} & 0 \\
0 & \frac{1}{\omega+\xi_{\boldsymbol{k}, \beta}}
\end{array}\right), \\
& g_{\mathrm{S}}(\boldsymbol{k}, \omega)=\left(\begin{array}{cc}
\frac{u_{\boldsymbol{k}}^{2}}{\omega-E_{\boldsymbol{k}}}+\frac{v_{\boldsymbol{k}}^{2}}{\omega+E_{\boldsymbol{k}}} & \frac{-u_{\boldsymbol{k}} v_{\boldsymbol{k}}}{\omega-E_{\boldsymbol{k}}}+\frac{u_{\boldsymbol{k}} v_{\boldsymbol{k}}}{\omega+E_{\boldsymbol{k}}} \\
\frac{-u_{\boldsymbol{k}} v_{\boldsymbol{k}}}{\omega-E_{\boldsymbol{k}}}+\frac{u_{\boldsymbol{k}} v_{\boldsymbol{k}}}{\omega+E_{\boldsymbol{k}}} & \frac{u_{\boldsymbol{k}}^{2}}{\omega+E_{\boldsymbol{k}}}+\frac{v_{\boldsymbol{k}}^{2}}{\omega-E_{\boldsymbol{k}}}
\end{array}\right) .
\end{aligned}
$$

The quasiparticle energies $E_{\boldsymbol{k}}=\sqrt{\xi_{\mathrm{S}, \boldsymbol{k}}^{2}+\Delta^{2}}$ have a well known gaped character and the BCS coefficients are given by $u_{\boldsymbol{k}}^{2}, v_{\boldsymbol{k}}^{2}=\frac{1}{2}\left[1 \pm \frac{\xi_{k s}}{E_{\boldsymbol{k}}}\right], u_{\boldsymbol{k}} v_{\boldsymbol{k}}=\frac{\Delta}{2 E_{\boldsymbol{k}}}$. In the wide band limit approximation we assume the constant hybridization couplings $\Gamma_{\beta}=2 \pi \sum_{\boldsymbol{k}}\left|V_{\beta, \boldsymbol{k}}\right|^{2} \Delta\left(\omega-\xi_{\beta \boldsymbol{k}}\right)$. We thus obtain the selfenergies

$$
\begin{aligned}
& \sum_{\boldsymbol{k}} V_{\mathrm{N}, \boldsymbol{k}} g_{\mathrm{N}}(\boldsymbol{k}, \omega) V_{\mathrm{N} \boldsymbol{k}}^{*}=-\mathrm{i} \frac{\Gamma_{\mathrm{N}}}{2}\left(\begin{array}{ll}
1 & 0 \\
0 & 1
\end{array}\right), \\
& \sum_{\boldsymbol{k}} V_{\mathrm{S}, \boldsymbol{k}} g_{\beta}(\boldsymbol{k}, \omega) V_{\mathrm{S}, \boldsymbol{k}}^{*}=-\mathrm{i} \frac{\Gamma_{\mathrm{S}}}{2} \gamma(\omega)\left(\begin{array}{cc}
1 & \frac{\Delta}{\omega} \\
\frac{\Delta}{\omega} & 1
\end{array}\right),
\end{aligned}
$$

where

$$
\gamma(\omega)=\frac{|\omega| \Theta(|\omega|-\Delta)}{\sqrt{\omega^{2}-\Delta^{2}}}-i \frac{\omega \Theta(\Delta-|\omega|)}{\sqrt{\Delta^{2}-\omega^{2}}} .
$$

In the remaining part of this work we will focus on a deep subgap regime $|\omega| \ll \Delta$ when (9) simplifies to the following static value:

$$
\sum_{\boldsymbol{k}} V_{\mathrm{S}, \boldsymbol{k}} g_{\beta}(\boldsymbol{k}, \omega) V_{\mathrm{S}, \boldsymbol{k}}^{*}=-\frac{\Gamma_{S}}{2}\left(\begin{array}{ll}
0 & 1 \\
1 & 0
\end{array}\right) .
$$

We now calculate the Green function $g_{\text {mol }}(\omega)$ of a vibrating quantum dot. For this purpose we use the canonical transformation to eliminate electron-phonon coupling term from Hamiltonian (2). Using the Lang-Firsov transformation $\mathrm{e}^{S} H_{\mathrm{QD}} \mathrm{e}^{-S}$ with generating operator [10]:

$$
\widehat{S}=\sum_{\sigma} d_{\sigma}^{\dagger} d_{\sigma} \frac{\lambda}{\omega_{0}}\left(a^{\dagger}-a\right),
$$

one obtains $\mathrm{e}^{S} H_{\mathrm{QD}} \mathrm{e}^{-S}=\sum_{\sigma} \tilde{\epsilon} \tilde{d}_{\sigma}^{\dagger} \tilde{d}_{\sigma}+\tilde{\omega}_{0} a^{\dagger} a+\tilde{U} \tilde{n}_{\downarrow} \tilde{n}_{\uparrow}$. The fermion and boson subsystems are thus effectively disentangled, renormalizing the energy $\tilde{\epsilon}=\epsilon-\Delta_{\mathrm{ph}}$ and the electron-electron potential $\tilde{U}=U-2 \Delta_{\mathrm{ph}}$ though the polaron shift $\Delta_{p h}=\lambda^{2} / \omega_{0}$. Additionally, the creation and annihilation operators are dressed

$$
\tilde{d}_{\sigma}^{(\dagger)}=d_{\sigma}^{(\dagger)} X^{(\dagger)}, \quad \tilde{a}^{(\dagger)}=a^{(\dagger)}-\frac{\lambda}{\omega_{0}} \sum_{\sigma} d_{\sigma}^{\dagger} d_{\sigma},
$$

where the polaronic cloud operator $X$ is defined as:

$$
X=\mathrm{e}^{-\left(\lambda / \omega_{0}\right)\left(a^{\dagger}-a\right)} .
$$

In order to calculate $g_{\mathrm{mol}}\left(t, t^{\prime}\right)$ we can use the following identities:

$$
\begin{aligned}
& g_{\mathrm{mol}}^{11}\left(t, t^{\prime}\right)=-\mathrm{i}\left\langle\widehat{T} d_{\sigma}(t) d_{\sigma}^{\dagger}\left(t^{\prime}\right) \mathrm{e}^{-S} \mathrm{e}^{S}\right\rangle= \\
& \quad-\mathrm{i}\left\langle\widehat{T} \mathrm{e}^{S} d_{\sigma}(t) d_{\sigma}^{\dagger}\left(t^{\prime}\right) \mathrm{e}^{-S}\right\rangle=-\mathrm{i}\left\langle\widehat{T} \tilde{d}_{\sigma}(t) \tilde{d}_{\sigma}^{\dagger}\right\rangle=
\end{aligned}
$$

$$
\begin{aligned}
& -\mathrm{i}\left\langle\widehat{T} d_{\sigma}(t) X(t) d_{\sigma}^{\dagger}\left(t^{\prime}\right) X^{\dagger}\left(t^{\prime}\right)\right\rangle= \\
& -\mathrm{i}\left\langle\widehat{T} d_{\sigma}(t) d_{\sigma}^{\dagger}\left(t^{\prime}\right)\right\rangle_{\mathrm{el}}\left\langle\widehat{T} X(t) X^{\dagger}\left(t^{\prime}\right)\right\rangle_{\text {vib }}= \\
& \tilde{G}_{\mathrm{el}}^{11}\left(t, t^{\prime}\right)\left\langle\widehat{T} X(t) X^{\dagger}\left(t^{\prime}\right)\right\rangle_{\text {vib }},
\end{aligned}
$$

where $\widehat{T}$ is the time ordering operator. Time dependence of the polaron cloud operator can be expressed by $X(t)=\mathrm{e}^{\mathrm{i} \omega_{0} a^{\dagger} a t} X \mathrm{e}^{\mathrm{i} \omega_{0} a^{\dagger} a t}$. After lengthy but straightforward algebra we finally obtain

$$
\begin{aligned}
& \left\langle X(t) X\left(t^{\prime}\right)^{\dagger}\right\rangle=\exp \left(-\left(\lambda / \omega_{0}\right)^{2}\left(\left(1-\mathrm{e}^{-\mathrm{i} \omega_{0}\left(t-t^{\prime}\right)}\right)\right.\right. \\
& \left.\left.\quad \times\left(1+N_{p}\right)+\left(1-\mathrm{e}^{\mathrm{i} \omega_{0}\left(t-t^{\prime}\right)}\right) N_{p}\right)\right)
\end{aligned}
$$

with the Bose-Einstein distribution $N_{p}=\left[\mathrm{e}^{\beta \omega_{0}}-1\right]^{-1}$. We now put this result into Eq. (14) and compute its Fourier transform assuming the equilibrium conditions, when the Green functions depend only on the time difference $t-t^{\prime}$. In such conditions [11]:

$$
\begin{gathered}
g_{\mathrm{mol}}^{11}(\omega)=\mathrm{e}^{-\left(\lambda \sqrt{1+2 N_{p}} / \omega_{0}\right)^{2}} \sum_{l} I_{l} \mathrm{e}^{l \beta \omega_{0} / 2} \tilde{G}\left(\omega-l \omega_{0}\right) \\
\times\left[2\left(\frac{\lambda}{\omega_{0}}\right)^{2} \sqrt{N_{p}\left(1+N_{p}\right)}\right],
\end{gathered}
$$

where $I_{l}$ are the modified Bessel functions. The Green function $g_{\mathrm{mol}}(\omega)$ contains both the electronic and bosonic degrees of freedom. Note that argument of the electronic Green function $\tilde{G}_{e l}$ depends on the number $l=0,1,2 \ldots$ of phonon quanta. At zero temperature this Eq. (16) simplifies to

$$
\lim _{T \rightarrow 0} g_{\mathrm{mol}}^{11}(\omega)=\sum_{l} \mathrm{e}^{-g} \frac{g^{l}}{l !} \tilde{G}_{e l}\left(\omega-l \omega_{0}\right),
$$

where $g=\left(\lambda / \omega_{0}\right)^{2}$ is a dimensionless adiabacity parameter. Neglecting the electron-electron interactions we can obtain the spectral function of a molecular quantum dot $\rho_{\text {mol }}(\omega)=-\pi^{-1} \operatorname{Im} g_{\text {mol }}^{11}\left(\omega+\mathrm{i} 0^{+}\right)$. At zero temperature this spectral function

$$
\rho_{\mathrm{mol}}(\omega)=\mathrm{e}^{-g} \sum_{l} \frac{g^{l}}{l !} \Delta\left(\omega-\tilde{\epsilon}-l \omega_{0}\right) .
$$

consists of the Dirac delta functions separated by the phonon energy $\omega_{0}$. Their amplitudes are expressed by the envelope factor $\mathrm{e}^{-g \frac{g^{l}}{l !}}[10,11]$. Since the matrix Green function $g_{\mathrm{mol}}(\omega)$ of an isolated molecular dot does not have any off-diagonal terms we finally get

$$
g_{\mathrm{mol}}(\omega)=\left(\begin{array}{cc}
g_{\mathrm{mol}}^{11}(\omega) & 0 \\
0 & \left.-\left[g_{\mathrm{mol}}^{11}(-\omega)\right]^{*}\right]
\end{array}\right) .
$$

\section{Proximity vs. polaron effects}

From Eq. (18) we can see that the electron-phonon interaction induces a series of phonon states starting from $\tilde{\epsilon}$. The initial single level spectrum is thus transformed into a multilevel structure (Fig. 1), where the lowest state is shifted by the polaronic term $\Delta_{\mathrm{ph}}$. These polaronic states are separated by the phonon energy $\omega_{0}$ whereas their amplitudes strongly depend on the coupling strength $\lambda[10]$. We shall now inspect how the 


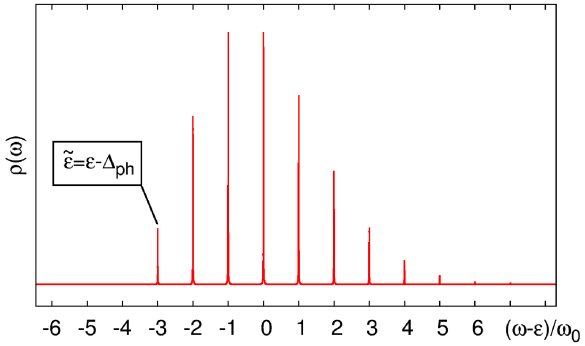

Fig. 1. Spectrum of a vibrating quantum dot obtained at zero temperature for $\lambda=3 \omega_{0}$ assuming the infinitesimally small $\Gamma_{\mathrm{N}}$. Energies are plotted with respect to initial QD level $\epsilon$.

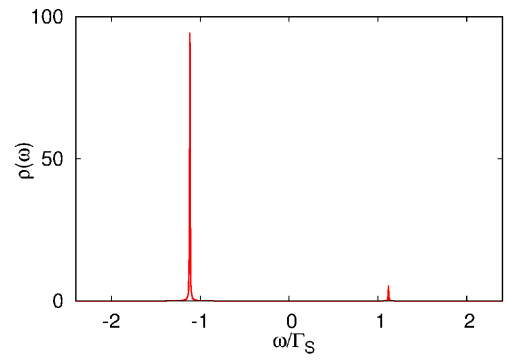

Fig. 2. Spectrum of the quantum dot coupled to the superconducting lead in absence of bosonic field. The result is obtained for the energy level $\epsilon=-\Gamma_{\mathrm{S}}$ and infinitesimally small $\Gamma_{\mathrm{N}}$.

induced on-dot pairing (due to $\Gamma_{\mathrm{S}}$ ) and the hybridization $\Gamma_{\mathrm{N}}$ with the normal lead affect these polaronic states.

We are now ready to combine the polaronic function (19) with the hybridization effects (8), (10). The effective spectral function $\rho(\omega)=-\pi^{-1} \operatorname{Im} G\left(\omega+\mathrm{i} 0^{+}\right)$can be easily determined from the Dyson Eq. (4).

In the previous works $[8,9]$ we have discussed in detail the influence of the proximity effect on the energy spectrum of single level quantum dot. Let us recall here some main conclusions of these studies, that are crucial for understanding the present problem. Due to the proximity effect the energy level $\epsilon$ (in the subgap regime) is split to the new quasiparticle energies $\pm E_{d}= \pm \sqrt{\epsilon^{2}+\left(\Gamma_{\mathrm{S}} / 2\right)^{2}}$ (see Fig. 2). Amplitudes of such (Andreev) states are given by the BCS coefficients $u_{d}^{2}, v_{d}^{2}=\frac{1}{2}\left[1 \pm \frac{\epsilon}{E_{d}}\right]$. Additional coupling $\Gamma_{\mathrm{N}}$ of the proximized quantum dot to the

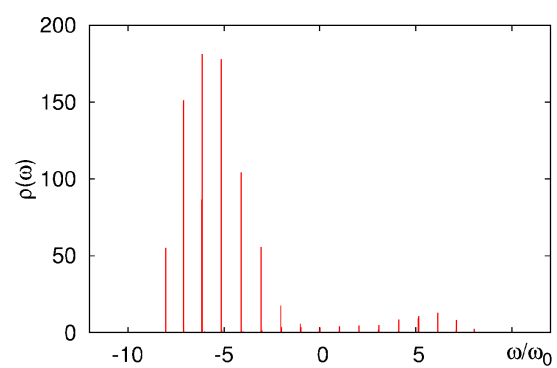

Fig. 3. Spectral function of a vibrating quantum dot obtained for $-\epsilon=\Gamma_{\mathrm{S}}=5 \omega_{0}$ and $\lambda=3 \omega_{0}$ and $\Gamma_{\mathrm{N}} / \Gamma_{\mathrm{S}}=$ $10^{-5}$.

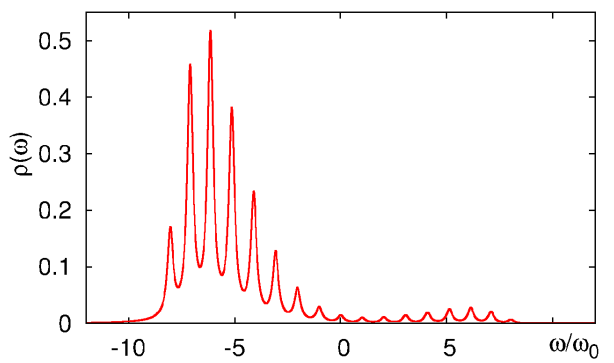

Fig. 4. Density of states of a vibrating quantum dot coupled to the superconducting lead. The results are obtained for similar parameters as in Fig. 3 with different $\Gamma_{\mathrm{N}}=\omega_{0}$.

metallic electrode causes a broadening of these Andreev quasiparticle states (i.e. a finite life-time effect).

In a case of the vibrating molecule the multiple levels driven by electron-phonon interaction $\lambda \neq 0$ appear simultaneously above and below Fermi surface. The lowest Andreev state is formed at the energy $-\tilde{E}_{d}=$ $-\sqrt{\tilde{\epsilon}^{2}+\left(\Gamma_{\mathrm{S}} / 2\right)^{2}}$. Other polaronic states appear at $\pm\left(\tilde{E}_{d}+l \omega_{0}\right)$ and amplitudes of these peaks depend both, on the factor $\mathrm{e}^{-g \frac{g^{l}}{l !}}$ and the BCS coefficients $\tilde{u}^{2}, \tilde{v}^{2}=$ $\frac{1}{2}\left[1 \pm \frac{\tilde{\epsilon}}{E_{d}}\right]$ (Fig. 3). Hybridization $\Gamma_{\mathrm{N}}$ is responsible for broadening these polaronic states (see Fig. 4) leading to a finite-life time of the subgap states.

\section{Summary}

We have shown that the electron-phonon interaction combined with the proximity effect induce a series of polaronic states simultaneously in the particle and hole excitations. Hybridization $\Gamma_{\mathrm{N}}$ with the metallic lead merely broadens these polaronic subgap states. In consequence at the sufficiently large ratio $\Gamma_{\mathrm{N}} \geq \omega_{0}$, the polaronic states eventually overlap with each other forming a bandlike spectrum.

\section{References}

[1] A.H. Dayem, R.J. Martin, Phys. Rev. Lett. 8, 246 (1962).

[2] G. Platero, R. Aguado, Phys. Rep. 395, 1 (2004).

[3] M. Galperin, M.A. Ratner, A. Nitzan, J. Phys. Condens. Matter 19, 103201 (2007).

[4] S. De Franceschi, L. Kouwenhoven, C. Schönenberger, W. Wernsdorfer, Nature Nanotechnol. 5, 703 (2010).

[5] B.H. Wu, J.C. Cao, C. Timm, Phys. Rev. B 86, 035406 (2012).

[6] S.-N. Zhang, W. Pei, T.-F. Fan, Q.-F. Sun, Phys. Rev. B 86, 104513 (2012).

[7] B. Dong, G.H. Ding, X.L. Lei, Phys. Rev. B 88, 075414 (2013).

[8] T. Domański, A. Donabidowicz, Phys. Rev. B 78, 073105 (2008).

[9] J. Barański, T. Domański, J. Phys. Condens. Matter 25, 435305 (2013).

[10] G.D. Mahan, Many-Particle Physics, Plenum, New York 2000. 
[11] J. Fransson, Non-Equilibrium Nano-Physics: A Many-Body Approach, Lect. Notes Phys., Vol. 809, Springer, Dordrecht 2010. 